International Journal of Mathematical Analysis

Vol. 9, 2015, no. 2, 61 - 72

HIKARI Ltd, www.m-hikari.com

http://dx.doi.org/10.12988/ijma.2015.411339

\title{
Constrained Ultraspherical-Weighted Orthogonal Polynomials on Triangle
}

\author{
Mohammad A. AlQudah \\ Department of Mathematics \\ Northwood University \\ Midland, MI 48640, USA
}

Copyright (C) 2014 Mohammad A. AlQudah. This is an open access article distributed under the Creative Commons Attribution License, which permits unrestricted use, distribution, and reproduction in any medium, provided the original work is properly cited.

\begin{abstract}
We construct Ultraspherical-weighted orthogonal polynomials $\mathscr{C}_{n, r}^{(\lambda, \gamma)}(u, v, w), \lambda>-\frac{1}{2}, \gamma>-1$, on the triangular domain $T$, where $2 \lambda+\gamma=1$. We show $\mathscr{C}_{n, r}^{(\lambda, \gamma)}(u, v, w), r=0,1, \ldots, n ; n \geq 0$ form an orthogonal system over the triangular domain $T$ with respect to the Ultraspherical weight function.
\end{abstract}

Mathematics Subject Classification: 33C45, 42C05, 33C70

Keywords: Ultraspherical, Orthogonal Polynomials, Bivariate, Triangular Domains

\section{Introduction}

Recent years have seen a great deal in the field of orthogonal polynomials, the Ultraspherical orthogonal polynomials are Amongst these polynomials [1, $2,10,13,20]$. Although the main definitions and properties were considered many years ago, the cases of two or more variables of orthogonal polynomials on triangular domains have been studied by few researchers $[11,12,19]$. Proriol [15] introduced the definition of the bivariate orthogonal polynomials on the triangle, and the results were summarized by C.F. Dunkl and T. Koornwinder $[5,11]$. 
Orthogonal polynomials with Ultraspherical weight function $\mathrm{W}^{(\lambda, \gamma)}(u, v, w)=$ $u^{\lambda-\frac{1}{2}} v^{\lambda-\frac{1}{2}}(1-w)^{\gamma}, \lambda>\frac{-1}{2}, \gamma>-1$ on triangular domain $T$ are defined in many articles and textbooks, for instance [3, 10]. These polynomials $C_{n, r}^{(\lambda, \gamma)}(u, v, w)$, are orthogonal to each polynomial of degree less than or equal to $n-1$, with respect to the defined weight function $\mathrm{W}^{(\lambda, \gamma)}(u, v, w)$ on $T$. However, for $r \neq s$, $C_{n, r}^{(\lambda, \gamma)}(u, v, w)$ and $C_{n, s}^{(\lambda, \gamma)}(u, v, w)$ are not orthogonal with respect to the weight function $\mathrm{W}^{(\lambda, \gamma)}(u, v, w)$ on $\mathrm{T}$.

S. Waldron start the work of a generalized beta integral and the limit of the Bernstein-Durrmeyer operator with Jacobi weights. Also, he computed orthogonal polynomials on a triangle by degree raising. Farouki [7] defined the orthogonal polynomials with respect to the weight function $\mathrm{W}(u, v, w)=1$ on a triangular domain $T$. These polynomials $P_{n, r}(u, v, w)$ defined in [7], are orthogonal to each polynomial of degree $\leq n-1$ and also orthogonal to each polynomial $P_{n, s}(u, v, w), r \neq s$.

In this paper, we construct orthogonal polynomials $\mathscr{C}_{n, r}^{(\lambda, \gamma)}(u, v, w)$, with respect to the Ultraspherical weight function $\mathrm{W}^{(\lambda, \gamma)}(u, v, w)=u^{\lambda-\frac{1}{2}} v^{\lambda-\frac{1}{2}}(1-$ $w)^{\gamma}, \lambda>\frac{-1}{2}, \gamma>-1$, on triangular domain $T$, such that $2 \lambda+\gamma=1$. These Ultraspherical-weighted orthogonal polynomials are given in terms of Bernstein basis, so many geometric properties of the Bernstein polynomial basis are preserve. We show that these bivariate polynomials $\mathscr{C}_{n, r}^{(\lambda, \gamma)}(u, v, w), r=0,1, \ldots, n$, and $n=0,1,2, \ldots$, form an orthogonal system over the triangular domain $T$ with respect to the weight function $\mathrm{W}^{(\lambda, \gamma)}(u, v, w)=u^{\lambda-\frac{1}{2}} v^{\lambda-\frac{1}{2}}(1-w)^{\gamma}, \lambda>$ $\frac{-1}{2}, \gamma>-1$, where $2 \lambda+\gamma=1$.

On the triangular domain $T$, we proved that these polynomials $\mathscr{C}_{n, r}^{(\lambda, \gamma)}(u, v, w) \in$ $\mathfrak{L}_{\mathfrak{n}}, n \geq 1, r=0,1, \ldots, n$, and for $r \neq s, \mathscr{C}_{n, r}^{(\lambda, \gamma)}(u, v, w) \perp \mathscr{C}_{n, s}^{(\lambda, \gamma)}(u, v, w)$.

P.K. Suetin [19] constructed bivariate orthogonal polynomials on the square. He considered the tensor product of the set of orthogonal polynomials over the domain $G=\{(x, y):-1 \leq x \leq 1,-1 \leq y \leq 1\}$.

Let $\left\{C_{n}^{\left(\lambda_{1}\right)}(x)\right\},\left\{Q_{m}^{\left(\lambda_{2}\right)}(y)\right\}$ be the Ultraspherical polynomials over $[-1,1]$ with respect to the weight functions $\mathrm{W}_{1}(x)=\left(1-x^{2}\right)^{\lambda_{1}-\frac{1}{2}}$, and $\mathrm{W}_{2}(y)=$ $\left(1-y^{2}\right)^{\lambda_{2}-\frac{1}{2}}$ respectively. P.K. Suetin [19], defined the bivariate polynomials $\left\{R_{n m}(x, y)\right\}$ on $G$ formed by the tensor products of the Ultraspherical polynomials by

$$
R_{n m}(x, y):=C_{n-m}^{\left(\lambda_{1}\right)}(x) Q_{m}^{\left(\lambda_{2}\right)}(y), n=0,1,2, \ldots, m=0,1, \ldots, n
$$

Then $\left\{R_{n m}(x, y)\right\}$ are orthogonal on the square $G$ with respect to the weight function $\mathrm{W}(x, y)=\mathrm{W}_{1}^{\left(\lambda_{1}\right)}(x) \mathrm{W}_{2}^{\left(\lambda_{2}\right)}(y)$. However, The construction of orthogonal polynomials over a triangular domain is not straightforward like the tensor product over the square. 


\section{Barycentric, and Bernstein Polynomials}

Consider a base triangle in the plane with the vertices $\mathbf{p}_{k}=\left(x_{k}, y_{k}\right), k=1,2,3$. Then every point $\mathbf{p}$ inside the triangle

$$
T=\{(u, v, w): u, v, w \geq 0, u+v+w=1\},
$$

can be written using the barycentric coordinates $(u, v, w)$, as $\mathbf{p}=u \mathbf{p}_{1}+v \mathbf{p}_{2}+$ $w \mathbf{p}_{3}$. The barycentric coordinates are given in the following ratios:

$$
u=\frac{\operatorname{area}\left(\mathbf{p}, \mathbf{p}_{2}, \mathbf{p}_{3}\right)}{\operatorname{area}\left(\mathbf{p}_{1}, \mathbf{p}_{2}, \mathbf{p}_{3}\right)}, \quad v=\frac{\operatorname{area}\left(\mathbf{p}_{1}, \mathbf{p}, \mathbf{p}_{3}\right)}{\operatorname{area}\left(\mathbf{p}_{1}, \mathbf{p}_{2}, \mathbf{p}_{3}\right)}, \quad w=\frac{\operatorname{area}\left(\mathbf{p}_{1}, \mathbf{p}_{2}, \mathbf{p}\right)}{\operatorname{area}\left(\mathbf{p}_{1}, \mathbf{p}_{2}, \mathbf{p}_{3}\right)}
$$

where area $\left(\mathbf{p}_{1}, \mathbf{p}_{2}, \mathbf{p}_{3}\right) \neq 0$, which means that $\mathbf{p}_{1}, \mathbf{p}_{2}, \mathbf{p}_{3}$ are not collinear.

Let the notation $\zeta=(i, j, k)$ denote triples of nonnegative integers, where $|\zeta|=i+j+k$. The generalized Bernstein polynomials of degree $n$ on the triangular domain $T$ are defined by the formula

$$
b_{\zeta}^{n}(u, v, w)=\left(\begin{array}{l}
n \\
\zeta
\end{array}\right) u^{i} v^{j} w^{k}, \quad|\zeta|=n, \quad \text { where }\left(\begin{array}{l}
n \\
\zeta
\end{array}\right)=\frac{n !}{i ! j ! k !} .
$$

Note that the generalized Bernstein polynomials are nonnegative over $T$, and form a partition of unity,

$$
1=(u+v+w)^{n}=\sum_{\substack{0 \leq i, j, k \leq n \\ i+j+k=n}} \frac{n !}{i ! j ! k !} u^{i} v^{j} w^{k} .
$$

These polynomials define the Bernstein basis for the space $\Pi_{n}$ over the triangular domain $T$, where the $k$ th row contains $k+1$ polynomials. Thus, for a basis of linearly independent polynomials of total degree $n$, there are a total of $(1 / 2)(n+1)(n+2)$ linearly independent polynomials.

Any polynomial $p(u, v, w)$ of degree $n$ can be written in the Bernstein form as

$$
p(u, v, w)=\sum_{|\zeta|=n} d_{\zeta} b_{\zeta}^{n}(u, v, w)
$$

with Bézier coefficients $d_{\zeta}$. We can also use the degree elevation algorithm for the Bernstein representation (1). This is obtained by multiplying both sides by $1=u+v+w$, and writing

$$
p(u, v, w)=\sum_{|\zeta|=n+1} d_{\zeta}^{(1)} b_{\zeta}^{n+1}(u, v, w),
$$

the new coefficients $d_{\zeta}^{(1)}$ are defined by, see $[6,9]$,

$$
d_{i, j, k}^{(1)}=\frac{1}{n+1}\left(i d_{i-1, j, k}+j d_{i, j-1, k}+k d_{i, j, k-1}\right), \quad i+j+k=n+1 .
$$


The Bernstein polynomials $b_{\zeta}^{n}(u, v, w),|\zeta|=n$, on $T$ satisfy, see [7],

$$
\iint_{T} b_{\zeta}^{n}(u, v, w) d A=\frac{\Delta}{(n+1)(n+2)},
$$

where $\Delta$ is double the area of $T$.

Let $p(u, v, w)$ and $q(u, v, w)$ be two bivariate polynomials over $T$, then we define their inner product over $T$ by

$$
\langle p, q\rangle=\frac{1}{\Delta} \iint_{T} p q d A .
$$

We say that $p$ and $q$ are orthogonal if $\langle p, q\rangle=0$.

For $m \geq 1$, we define $\mathfrak{L}_{\mathfrak{m}}=\left\{p \in \Pi_{m}: p \perp \Pi_{m-1}\right\}$ to be the space of polynomials of degree $m$ that are orthogonal to all polynomials of degree $<m$ over a triangular domain $T$, and $\Pi_{n}$ is the space of all polynomials of degree $n$ over the triangular domain $T$.

Let $f(u, v, w)$ be an integrable function over $T$ and consider the operator

$$
S_{n}(f)=(n+1)(n+2) \sum_{|\zeta|=n}\left\langle f, b_{\zeta}^{n}\right\rangle b_{\zeta}^{n} .
$$

For $n \geq m$

$$
\lambda_{m, n}=\frac{(n+2) ! n !}{(n+m+2) !(n-m) !}
$$

is an eigenvalue of the operator $S_{n}$ and $\mathfrak{L}_{\mathfrak{m}}$ is the corresponding eigenspace, see [4] for proof and more details. The following lemmas will be needed in the proof of the main results, see $[7,14]$ for the proofs and more details.

Lemma 2.1. (See [7]). Let $p=\sum_{|\zeta|=n} c_{\zeta} b_{\zeta}^{n} \in \mathfrak{L}_{\mathfrak{m}}$ and let $q=\sum_{|\zeta|=n} d_{\zeta} b_{\zeta}^{n} \in$ $\Pi_{n}$ with $m \leq n$. Then,

$$
\langle p, q\rangle=\frac{(n !)^{2}}{(n+m+2) !(n-m) !} \sum_{|\zeta|=n} c_{\zeta} d_{\zeta}
$$

Lemma 2.2. (See [14]). Let $p \in \sum_{|\zeta|=n} c_{\zeta} b_{\zeta}^{n} \in \mathfrak{L}_{\mathfrak{n}}$. Then,

$$
p \in \mathfrak{L}_{\mathfrak{n}} \Longleftrightarrow \sum_{|\zeta|=n} c_{\zeta} d_{\zeta}=0 \quad \forall q=\sum_{|\zeta|=n} d_{\zeta} b_{\zeta}^{n} \in \Pi_{n-1} .
$$

\section{Ultraspherical Polynomials}

The Ultraspherical polynomials $C_{n}^{(\lambda)}(x)$ of degree $n$ are the orthogonal polynomials, except for a constant factor, on $[-1,1]$ with respect to the weight function

$$
\mathrm{W}(x)=\left(1-x^{2}\right)^{\lambda-\frac{1}{2}}, \lambda>-\frac{1}{2}
$$


In this paper, it is appropriate to take $x \in[0,1]$ for both Bernstein and Ultraspherical polynomials.

The following lemmas, See A. Rababah [17], will be needed in the construction of the orthogonal bivariate polynomials and the proof of the main results. For more details and the proofs, see [17]. Although the Pochhammer symbol is more appropriate, the combinatorial notation will be used, Szegö [20], since it is more compact and readable formulas.

Lemma 3.1. The Ultraspherical polynomials $C_{r}^{(\lambda)}(x)$ have the Bernstein representation:

$$
C_{r}^{(\lambda)}(x)=\frac{\left(\lambda+\frac{1}{2}\right)_{n}}{(2 \lambda)_{n}} \sum_{i=0}^{r}(-1)^{r-i} \frac{\left(\begin{array}{c}
r+\lambda-\frac{1}{2} \\
i
\end{array}\right)\left(\begin{array}{c}
r+\lambda-\frac{1}{2} \\
r-i
\end{array}\right)}{\left(\begin{array}{l}
r \\
i
\end{array}\right)} b_{i}^{r}(x), r=0,1, \ldots
$$

Lemma 3.2. The Ultraspherical polynomials $C_{0}^{(\lambda)}(x), \ldots, C_{n}^{(\lambda)}(x)$ of degree $\leq n$ can be expressed in the Bernstein basis of fixed degree $n$ by the following formula

$$
C_{r}^{(\lambda)}(x)=\sum_{i=0}^{n} \mu_{i, r}^{n} b_{i}^{n}(x), \quad r=0,1, \ldots, n
$$

where

$\mu_{i, r}^{n}=\frac{\left(\lambda+\frac{1}{2}\right)_{n}}{(2 \lambda)_{n}}\left(\begin{array}{c}n \\ i\end{array}\right)^{-1} \sum_{k=\max (0, i+r-n)}^{\min (i, r)}(-1)^{r-k}\left(\begin{array}{c}n-r \\ i-k\end{array}\right)\left(\begin{array}{c}r+\lambda-\frac{1}{2} \\ k\end{array}\right)\left(\begin{array}{c}r+\lambda-\frac{1}{2} \\ r-k\end{array}\right)$

In addition, the following combinatorial identity, Lemma 3.3 [18], can be used for the main results simplifications.

Lemma 3.3. For an integer n, we have the following combinatorial identity

$$
\left(\begin{array}{c}
n-\frac{1}{2} \\
n-k
\end{array}\right)\left(\begin{array}{c}
n-\frac{1}{2} \\
k
\end{array}\right)=\frac{1}{2^{2 n}}\left(\begin{array}{c}
2 n \\
n
\end{array}\right)\left(\begin{array}{c}
2 n \\
2 k
\end{array}\right) .
$$

In the following lemma, let

$$
q_{n, r}(w)=\sum_{j=0}^{n-r}(-1)^{j}\left(\begin{array}{c}
n+r+1 \\
j
\end{array}\right) b_{j}^{n-r}(w) .
$$

Lemma 3.4. (See [7]). For $r=0, \ldots, n$ and $i=0, \ldots, n-r-1, q_{n, r}(w)$ is orthogonal to $(1-w)^{2 r+i+1}$ on $[0,1]$, and hence, for every polynomial $p(w)$ of degree $\leq n-r-1$,

$$
\int_{0}^{1} q_{n, r}(w) p(w)(1-w)^{2 r+1} d w=0 .
$$




\section{Ultraspherical-Weighted Polynomials}

Analogous to [7], a simple closed-form representation of degree-ordered system of orthogonal polynomials is constructed on a triangular domain $T$. Since the Bernstein polynomials are stable [8], it is convenient to write these polynomials in Bernstein form.

For $n=0,1,2, \ldots$ and $r=0,1, \ldots, n$ we define the bivariate polynomials

$$
\mathscr{C}_{n, r}^{(\lambda, \gamma)}(u, v, w)=\sum_{i=0}^{r} c(i, \lambda) b_{i}^{r}(u, v) \sum_{j=0}^{n-r}(-1)^{j}\left(\begin{array}{c}
n+r+1 \\
j
\end{array}\right) b_{j}^{n-r}(w, u+v)
$$

where $\lambda>-\frac{1}{2}, \gamma>-1,2 \lambda+\gamma=1, b_{i}^{r}(u, v)=\left(\begin{array}{c}r \\ i\end{array}\right) u^{i} v^{r-i}, \quad i=0,1, \ldots, r$, and

$$
c(i, \lambda)=(-1)^{r-i} \frac{\left(\begin{array}{c}
r+\lambda-\frac{1}{2} \\
i
\end{array}\right)\left(\begin{array}{c}
r+\lambda-\frac{1}{2} \\
r-i
\end{array}\right)}{\left(\begin{array}{c}
r \\
i
\end{array}\right)}, i=0,1, \ldots, r .
$$

In this section, we show that the polynomials $\mathscr{C}_{n, r}^{(\lambda, \gamma)}(u, v, w) \in \mathfrak{L}_{n}, r=$ $0,1, \ldots, n ; n \geq 1$, and for $r \neq s, \mathscr{C}_{n, r}^{(\lambda, \gamma)} \perp \mathscr{C}_{n, s}^{(\lambda, \gamma)}$. By choosing $\mathscr{C}_{0,0}^{(\lambda, \gamma)}=1$, the polynomials $\mathscr{C}_{n, r}^{(\lambda, \gamma)}(u, v, w)$ for $0 \leq r \leq n$ and $n \geq 0$ form a degree-ordered orthogonal sequence over $T$.

We first rewrite these polynomials in the Ultraspherical polynomials form:

$$
\begin{aligned}
\mathscr{C}_{n, r}^{(\lambda, \gamma)}(u, v, w) & =\sum_{i=0}^{r} c(i, \lambda) b_{i}^{r}(u, v) \sum_{j=0}^{n-r}(-1)^{j}\left(\begin{array}{c}
n+r+1 \\
j
\end{array}\right) b_{j}^{n-r}(w, u+v) \\
& =\sum_{i=0}^{r} c(i, \lambda) \frac{b_{i}^{r}(u, v)}{(u+v)^{r}}(1-w)^{r} \sum_{j=0}^{n-r}(-1)^{j}\left(\begin{array}{c}
n+r+1 \\
j
\end{array}\right) b_{j}^{n-r}(w, 1-w) .
\end{aligned}
$$

Since $b_{i}^{r}(u, v)=(u+v)^{r} b_{i}^{r}\left(\frac{u}{1-w}\right)$, and using Lemma 3.1 we get

$$
\mathscr{C}_{n, r}^{(\lambda, \gamma)}(u, v, w)=\frac{\left(\lambda+\frac{1}{2}\right)_{n}}{(2 \lambda)_{n}} C_{r}^{(\lambda)}\left(\frac{u}{1-w}\right)(1-w)^{r} q_{n, r}(w), \quad r=0, \ldots, n,
$$

where $C_{r}^{(\lambda)}(t)$ is the univariate Ultraspherical polynomial of degree $r$ and $q_{n, r}(w)$ is defined in equation (5).

For simplicity, since we are dealing with orthogonality, and the Ultraspherical polynomials $C_{n}(x)$ of degree $n$ are the orthogonal except for a constant factor, we rewrite (8) as

$$
\mathscr{C}_{n, r}^{(\lambda, \gamma)}(u, v, w)=C_{r}^{(\lambda)}\left(\frac{u}{1-w}\right)(1-w)^{r} q_{n, r}(w), \quad r=0, \ldots, n .
$$

First, we show that the polynomials $\mathscr{C}_{n, r}^{(\lambda, \gamma)}(u, v, w), r=0, \ldots, n$, are orthogonal to all polynomials of degree less than $n$ over the triangular domain T. 
Theorem 4.1. For each $n=1,2, \ldots, r=0,1, \ldots, n$, and the weight function $\mathrm{W}^{(\lambda, \gamma)}(u, v, w)=u^{\lambda-\frac{1}{2}} v^{\lambda-\frac{1}{2}}(1-w)^{\gamma}$ such that $\lambda>-\frac{1}{2}, \gamma>-1,2 \lambda+\gamma=1$, $\mathscr{C}_{n, r}^{(\lambda, \gamma)}(u, v, w) \in \mathfrak{L}_{n}$.

Proof. For each $m=0, \ldots, n-1$, and $s=0, \ldots, m$ we construct the set of bivariate polynomials

$Q_{s, m}^{(\lambda)}(u, v, w)=C_{s}^{(\lambda)}\left(\frac{u}{1-w}\right)(1-w)^{m} w^{n-m-1}, m=0, \ldots, n-1, s=0, \ldots, m$.

The span of these polynomials includes the set of Bernstein polynomials

$b_{j}^{m}\left(\frac{u}{1-w}\right)(1-w)^{m} w^{n-m-1}=b_{j}^{m}(u, v) w^{n-m-1} m=0, \ldots, n-1, j=0, \ldots, m$,

which span $\Pi_{n-1}$. Thus, it is sufficient to show that for each $m=0, \ldots, n-1$, $s=0, \ldots, m$, we have

$$
I:=\iint_{T} \mathscr{C}_{n, r}^{(\lambda, \gamma)}(u, v, w) Q_{s, m}^{(\lambda)}(u, v, w) \mathrm{W}^{(\lambda, \gamma)}(u, v, w) d A=0 .
$$

This is simplified to

$=\Delta \int_{0}^{1} \int_{0}^{1-w} C_{r}^{(\lambda)}\left(\frac{u}{1-w}\right) q_{n, r}(w) C_{s}^{(\lambda)}\left(\frac{u}{1-w}\right) w^{n-m-1} u^{\lambda-\frac{1}{2}} v^{\lambda-\frac{1}{2}}(1-w)^{\gamma+r+m} d u d w$.

By making the substitution $t=\frac{u}{1-w}$, we get

$$
\begin{aligned}
I & =\Delta \int_{0}^{1} \int_{0}^{1} C_{r}^{(\lambda)}(t) q_{n, r}(w) C_{s}^{(\lambda)}(t)(1-w)^{2 \lambda+\gamma+r+m} w^{n-m-1} t^{\lambda-\frac{1}{2}}(1-t)^{\lambda-\frac{1}{2}} d t d w \\
& =\Delta \int_{0}^{1} C_{r}^{(\lambda)}(t) C_{s}^{(\lambda)}(t) t^{\lambda-\frac{1}{2}}(1-t)^{\lambda-\frac{1}{2}} d t \int_{0}^{1} q_{n, r}(w)(1-w)^{2 \lambda+\gamma+r+m} w^{n-m-1} d w .
\end{aligned}
$$

If $m<r$, then we have $s<r$, and the first integral is zero by the orthogonality property of the Ultraspherical polynomials. If $r \leq m \leq n-1$, we have by Lemma 3.4 the second integral equals zero, Thus the theorem follows.

Note that taking $\mathrm{W}^{(\lambda, \gamma)}(u, v, w)=u^{\lambda-\frac{1}{2}} v^{\lambda-\frac{1}{2}}(1-w)^{\gamma}$ enables us to separate the integrand in the proof of Theorem 4.1. Also note that taking $2 \lambda+\gamma=1$ enables us to use Lemma 3.4 in the proof of Theorem 4.1.

In the following theorem, we show that $\mathscr{C}_{n, r}^{(\lambda, \gamma)}(u, v, w)$ is orthogonal to each polynomial of degree $n$. And thus the bivariate polynomials $\mathscr{C}_{n, r}^{(\lambda, \gamma)}(u, v, w), r=$ $0,1, \ldots, n$, and $n=0,1,2, \ldots$ form an orthogonal system over the triangular domain $T$ with respect to the weight function $\mathrm{W}^{(\lambda, \gamma)}(u, v, w), \lambda>-\frac{1}{2}, \gamma>-1$. 
Theorem 4.2. For $r \neq s$, we have $\mathscr{C}_{n, r}^{(\lambda, \gamma)}(u, v, w) \perp \mathscr{C}_{n, s}^{(\lambda, \gamma)}(u, v, w)$ with respect to the weight function $\mathrm{W}^{(\lambda, \gamma)}(u, v, w)=u^{\lambda-\frac{1}{2}} v^{\lambda-\frac{1}{2}}(1-w)^{\gamma}$ such that $\lambda>-\frac{1}{2}, \gamma>-1$.

Proof. For $r \neq s$, we have

$$
\begin{gathered}
I:=\iint_{T} \mathscr{C}_{n, r}^{(\lambda, \gamma)}(u, v, w) \mathscr{C}_{n, s}^{(\lambda, \gamma)}(u, v, w) \mathrm{W}^{(\lambda, \gamma)}(u, v, w) d A \\
=\Delta \int_{0}^{1} \int_{0}^{1-w} C_{r}^{(\lambda)}\left(\frac{u}{1-w}\right) C_{s}^{(\lambda)}\left(\frac{u}{1-w}\right)(1-w)^{r+s} q_{n, r}(w) q_{n, s}(w) \mathrm{W}^{(\lambda, \gamma)}(u, v, w) d u d w .
\end{gathered}
$$

By making the substitution $t=\frac{u}{1-w}$, we have

$$
I=\Delta \int_{0}^{1} C_{r}^{(\lambda)}(t) C_{s}^{(\lambda)}(t) t^{\lambda-\frac{1}{2}}(1-t)^{\lambda-\frac{1}{2}} d t \int_{0}^{1} q_{n, r}(w) q_{n, s}(w)(1-w)^{2 \lambda+\gamma+r+s} d w
$$

the first integral equals zero by orthogonality property of the Ultraspherical polynomials for $r \neq s$, and thus the theorem follows.

\section{Ultraspherical in Bernstein Basis}

The Bernstein-Bézier form of curves and surfaces exhibits some interesting geometric properties, see $[6,9]$. So, we write the orthogonal polynomials $\mathscr{C}_{n, r}^{(\lambda, \gamma)}(u, v, w), \quad r=0,1, \ldots, n$ and $n=0,1,2, \ldots$ in the following BernsteinBézier form:

$$
\mathscr{C}_{n, r}^{(\lambda, \gamma)}(u, v, w)=\sum_{|\zeta|=n} a_{\zeta}^{n, r} b_{\zeta}^{n}(u, v, w)
$$

We are interested in finding a closed form for the computation of the Bernstein coefficients $a_{\zeta}^{n, r}$. These are given explicitly in the following theorem:

Theorem 5.1. The Bernstein coefficients $a_{\zeta}^{n, r}$ of equation (13) are given explicitly by

$$
a_{i j k}^{n, r}= \begin{cases}(-1)^{k} \frac{\left(\begin{array}{c}
n+r+1 \\
k
\end{array}\right)\left(\begin{array}{c}
n-r \\
k
\end{array}\right)}{\left(\begin{array}{c}
n \\
k
\end{array}\right)} \mu_{i, r}^{n-k} & 0 \leq k \leq n-r \\
0 & k>n-r\end{cases}
$$

where $\mu_{i, r}^{n-k}$ are given in (4). 
Proof. From equation (6), it is clear that $\mathscr{C}_{n, r}^{(\lambda, \gamma)}(u, v, w)$ has degree $\leq n-r$ in the variable $w$, and thus

$$
a_{i j k}^{n, r}=0 \text { for } k>n-r .
$$

For $0 \leq k \leq n-r$, the remaining coefficients are determined by equating (6) and (13) as follows

$$
\sum_{i+j=n-k} a_{i j k}^{n, r} b_{i j k}^{n}(u, v, w)=(-1)^{k}\left(\begin{array}{c}
n+r+1 \\
k
\end{array}\right) b_{k}^{n-r}(w, u+v) \sum_{i=0}^{r} c(i, \lambda) b_{i}^{r}(u, v) .
$$

Comparing powers of $w$ on both sides, we have

$$
\sum_{i=0}^{n-k} a_{i j k}^{n, r} \frac{n !}{i ! j ! k !} u^{i} v^{j}=(-1)^{k}\left(\begin{array}{c}
n+r+1 \\
k
\end{array}\right)\left(\begin{array}{c}
n-r \\
k
\end{array}\right)(u+v)^{n-r-k} \sum_{i=0}^{r} c(i, \lambda) b_{i}^{r}(u, v) .
$$

The left hand side of the last equation can be written in the form

$$
\sum_{i=0}^{n-k} a_{i j k}^{n, r} \frac{n !(n-k) !}{i !(n-k-i) ! k !(n-k) !} u^{i} v^{j}=\sum_{i=0}^{n-k} a_{i j k}^{n, r}\left(\begin{array}{l}
n \\
k
\end{array}\right) b_{i}^{n-k}(u, v)
$$

Now, we get

$$
\sum_{i=0}^{n-k} a_{i j k}^{n, r}\left(\begin{array}{l}
n \\
k
\end{array}\right) b_{i}^{n-k}(u, v)=(-1)^{k}\left(\begin{array}{c}
n+r+1 \\
k
\end{array}\right)\left(\begin{array}{c}
n-r \\
k
\end{array}\right)(u+v)^{n-r-k} \sum_{i=0}^{r} c(i, \lambda) b_{i}^{r}(u, v) .
$$

With some binomial simplifications, and using Lemma 3.2, we get

$$
\sum_{i=0}^{n-k} a_{i j k}^{n, r}\left(\begin{array}{l}
n \\
k
\end{array}\right) b_{i}^{n-k}(u, v)=(-1)^{k}\left(\begin{array}{c}
n+r+1 \\
k
\end{array}\right)\left(\begin{array}{c}
n-r \\
k
\end{array}\right) \sum_{i=0}^{r} \mu_{i, r}^{n-k} b_{i}^{n-k}(u, v),
$$

where $\mu_{i, r}^{n-k}$ are the coefficients resulting from writing Ultraspherical polynomial of degree $r$ in the Bernstein basis of degree $n-k$, as defined by expression (4). Thus, the required Bernstein-Bézier coefficients are given by:

$$
a_{i j k}^{n, r}=\left\{\begin{array}{ll}
(-1)^{k} \frac{\left(\begin{array}{c}
n+r+1 \\
k
\end{array}\right)\left(\begin{array}{c}
n-r \\
k
\end{array}\right)}{\left(\begin{array}{l}
n \\
k
\end{array}\right)} \mu_{i, r}^{n-k} & 0 \leq k \leq n-r \\
0 & k>n-r
\end{array} .\right.
$$

To derive a recurrence relation for the coefficients $a_{i j k}^{n, r}$ of $\mathscr{C}_{n, r}^{(\lambda, \gamma)}(u, v, w)$, consider the generalized Bernstein polynomial of degree $n-1$,

$$
\begin{aligned}
b_{i j k}^{n-1}(u, v, w) & =\frac{(n-1) !}{i ! j ! k !} u^{i} v^{j} w^{k}(u+v+w) \\
& =\frac{(i+1)}{n} b_{i+1, j, k}^{n}(u, v, w)+\frac{(j+1)}{n} b_{i, j+1, k}^{n}(u, v, w)+\frac{(k+1)}{n} b_{i, j, k+1}^{n}(u, v, w)
\end{aligned}
$$


by construction of $\mathscr{C}_{n, r}^{(\lambda, \gamma)}(u, v, w)$, we have $\left\langle b_{i j k}^{n-1}(u, v, w), \mathscr{C}_{n, r}^{(\lambda, \gamma)}(u, v, w)\right\rangle=0$, $i+j+k=n-1$, and thus by Lemma 2.2 , we obtain

$$
(i+1) a_{i+1, j, k}^{n, r}+(j+1) a_{i, j+1, k}^{n, r}+(k+1) a_{i, j, k+1}^{n, r}=0 .
$$

But, form Theorem 5.1, we have

$$
a_{i, n-i, 0}^{n, r}=\mu_{i, r}^{n} \text { for } i=0,1, \ldots, n ;
$$

we can use (17) to generate $a_{i, j, k}^{n, r}$ recursively on $k$.

Acknowledgements. The author would like to thank the referee for his valuable comments and suggestions.

\section{References}

[1] M. Abramowitz, and I.A. Stegun (Eds.), Handbook of Mathematical Functions with Formulas, Graphs, and Mathematical Tables, 9th printing. New York: Dover, 1972.

[2] T.S. Chihara, An Introduction to Orthogonal Polynomials, Gordon and Breach, New York-London-Paris, 1978.

[3] C. de Boor, B-form basics. In G. Farin (editor) Geometric Modeling: Algorithms and New Trends, pp. 131-148, Philadelphia, SIAM, 1987.

[4] M.-M. Derriennic, On multivariate approximation by Bernsteintype polynomials, J. Approx. Theory, 45 (2) (1985), 155-166. http://dx.doi.org/10.1016/0021-9045(85)90043-7

[5] C. F. Dunkl, and Y. Xu, Orthogonal Polynomials of Several Variables, Encyclopedia of Mathematics and its Applications, vol. 81, Cambridge University Press, Cambridge, 2001. http://dx.doi.org/10.1017/cbo9780511565717

[6] G. Farin, Curves and Surface for Computer Aided Geometric Design, 3rd ed., Computer Science and Scientific Computing, Academic Press, Massachusetts, 1993.

[7] R. T. Farouki, T. N. T. Goodman and T. Sauer, Construction of orthogonal bases for polynomials in Bernstein form on triangular and simplex domains, Comput. Aided Geom. Design, 20 (2) (2003), 209 - 230. http://dx.doi.org/10.1016/s0167-8396(03)00025-6 
[8] R. T. Farouki and V. T. Rajan, On the numerical condition of polynomials in Bernstein form, Comput. Aided Geom. Design, 4 (3) (1987), 191-216. http://dx.doi.org/10.1016/0167-8396(87)90012-4

[9] J. Hoschek, and D. Lasser, Fundamentals of Computer Aided Geometric Design, A.K.Peters, Massachusetts, 1993.

[10] D.S. Kim, T. Kim and S.-H. Rim, Some identities involving Gegenbauer polynomials, Advances in Difference Equations, arXiv:1207.7165v1 [math.NT], (2012). http://dx.doi.org/10.1186/1687-1847-2012-219

[11] T. H. Koornwinder, Two-variable analogues of the classical orthogonal polynomials, in: Theory and application of special functions, R.A. Askey (ed.), Academic Press, 1975, pp. 435-495. http://dx.doi.org/10.1016/b978-0-12-064850-4.50015-x

[12] T. H. Koornwinder and S.A. Sauter, The intersection of bivariate orthogonal polynomials on triangle patches, accepted by Mathematics of Computation, arXiv:1307.8429v3 [math.NA], (2013).

[13] F. W. J. Olver, D. W. Lozier, R. F. Boisvert, and C. W. Clark (editors), NIST handbook of mathematical functions, Cambridge University Press, Cambridge, 2010.

[14] J. Peters and U. Reif, Least squares approximation of Bézier coefficients provides best degree reduction in the L2-norm, J. Approx. Theory, 104 (1) (2000), 90 - 97. http://dx.doi.org/10.1006/jath.1999.3440

[15] J. Proriol, Sur une famille de polynomes a deux variables orthogonaux dans un triangle, C. R. Acad. Sci. Paris, 245 (1957), 2459 - 2461.

[16] A. Rababah, Distance for degree raising and reduction of triangular Bézier surfaces, J. Comput. Appl. Math., 158 (2) (2003), 233 - 241. http://dx.doi.org/10.1016/s0377-0427(03)00432-1

[17] A. Rababah, Jacobi-Bernstein basis transformation, Comput. Methods Appl. Math., 4 (2) (2004), 206 - 214. http://dx.doi.org/10.2478/cmam2004-0012

[18] A. Rababah, Transformation of Chebyshev Bernstein polynomial basis, Comput. Methods Appl. Math., 3 (4) (2003), 608 - 622. http://dx.doi.org/10.2478/cmam-2003-0038

[19] P. K., Suetin, Orthogonal Polynomials in Two Variables, Nauka, Moscow, 1999. 
[20] G. Szegö, Orthogonal Polynomials, American Mathematical Society, 4th ed., Rhode Island, 1975.

Received: November 12, 2014; Published: January 9, 2015 